\title{
On Finite Rank Operators on Centrally Closed Semiprime Rings
}

\author{
J. C. Cabello' ${ }^{1}$, R. Casas ${ }^{2}$, P. Montiel ${ }^{3}$ \\ ${ }^{1}$ Departamento de Análisis Matemático, Facultad de Ciencias, Universidad de Granada, Granada, Spain \\ ${ }^{2}$ Departamento de Didáctica de las Ciencias Experimentales, Facultad de Ciencias de la Educación, Universidad \\ de Granada, Granada, Spain \\ ${ }^{3}$ Departamento de Matemáticas, Centro de Magisterio La Inmaculada, Universidad de Granada, Granada, Spain \\ Email: jcabello@ugr.es, ricardocasas@ugr.es, pamonti@correo.ugr.es
}

Received 2 July 2014; revised 2 August 2014; accepted 16 August 2014

Copyright (C) 2014 by authors and Scientific Research Publishing Inc.

This work is licensed under the Creative Commons Attribution International License (CC BY).

http://creativecommons.org/licenses/by/4.0/

(c) (i) Open Access

\section{Abstract}

We prove that the multiplication ring of a centrally closed semiprime ring $R$ has a finite rank operator over the extended centroid $C$ iff $R$ contains an idempotent $q$ such that $q R q$ is finitely generated over $C$ and, for each $x \in q R q$, there exist $z \in q R q$ and $e$ an idempotent of $C$ such that $x z=e q$.

\section{Keywords}

\section{Ring, Semiprime Ring, Extended Centroid, Minimal Idempotent}

\section{Introduction}

The symmetric ring of quotients $Q_{s}(R)$ of a semiprime ring $R$ is probably the most comfortable ring of quotients of $R$. This notion was first introduced by W.S. Martindale [1] for prime rings and extended to the semiprime case by Amitsur [2]. Recall that a ring $R$ is said to be semiprime (resp. prime) if $I^{2} \neq 0$ for every nonzero ideal $I$ of $R$ (resp. if $I J \neq 0$ for all nonzero ideals $I, J$ of $R$ ). The center $C$ of $Q_{s}(R)$ is called the extended centroid of $R$, and the $C$-subring $Q_{R}:=R C$ of $Q_{s}(R)$ generated by $R$ is called the central closure of $R$. A semiprime $R$ is said to be centrally closed whenever $R=R C$. For every $q \in R$, we will denote $L_{q}$ and $R_{q}$ the left and right multiplication operators, respectively, by $q$ on $R$. The multiplication ring of $R, M(R)$, is defined as the subring of $L(R)$ generated by the identity operator $I d_{R}$ and the set $\left\{L_{q}, R_{q} \mid q \in R\right\}$. The goal of this paper is to give a semiprime extension of the following well-known result (see for instance [3], Theorem A.9):

"If the multiplication ring of a centrally closed prime ring $R$ has a finite rank operator over $C$ then $R$ contains an idempotent $q$ such that $q R q$ is a division algebra finitely generated over $C$ ". 
It is also well know that the extended centroid of a prime ring is a field, however, for a semiprime ring, we can only assert that said extended centroid is a von Neumann regular ring. This is the cause of the difficulty of extending this result. The starting point of this path relies on the fact that each subset $S$ of $Q_{S}(R)$ has an associated idempotent $e_{[s]}$ of the extended centroid $C$ (see [4], Theorem 2.3.9) and on a consequence (see [4], Theorem 2.3.3 and Proposition 1.1 below) of the Weak Density Theorem ([4], Theorem 1.1.5).

\section{Tools}

We shall assume throughout this paper that $R$ is a centrally closed semiprime ring. First of all, we recall that if $\mathcal{B}_{R}$ is the set of all idempotents in $C$ has a partial order given by $e \leq f$ iff $e=e f$. Moreover, $\mathcal{B}_{R}$ is a Boolean algebra for the operations

$$
e \wedge f=e f, \quad e \vee f=e+f-e f, \quad \text { and } \quad e^{*}=1-e .
$$

In fact, [5], Theorem 1.8 remains valid in case that $A=R$ is a ring, and so this Boolean algebra is complete, that is, every subset of $\mathcal{B}_{R}$ admits supremum and infimum. We will use the properties of the idempotent associated to a subset referred to in ([4], Theorem 2.3.9 (i) and (ii)) without notice.

Given a $C$-submodule $M$ of $R$, we will say that $M$ is $C$-finitely generated if there exist $q_{1}, q_{2}, \cdots$, $q_{n} \in R$ such that $M \subseteq \sum_{i=1}^{n} C q_{i}$.

Next, we establish our main tool.

Proposition 1.1 Let $N$ be a $C$-finitely generated $C$-submodule of $R$, and let $q \in R$. Then there exists $f_{0} \in \mathcal{B}_{R}$ such that: a) $\left.f_{0} \leq e_{[N]}, b\right) \quad f_{0} q \in N$ and $\left.c\right) \quad N+C q=N \oplus\left(1-f_{0}\right) q$.

Proof. We denote $e=e_{[N]}$. If $q \in N$, then $f_{0}=e_{[N]}$. Suppose that $q \in R \backslash N$. If $N \cap C q=0$, then we take $f_{0}=0$. In other case, take $e \lambda q \in N \cap C q$, for some $\lambda \in C$. By ([4] Theorem 2.3.9), there exists $\mu \in C$ such that $\lambda \mu \lambda=\lambda$ and $\lambda \mu \in \mathcal{B}_{R}$. In particular, $\lambda \mu e q \in N$, and $\lambda \mu e \leq e$. Thus, the family $\left\{f_{i}\right\} \subseteq \mathcal{B}_{R}$ of all nonzero idempotents satisfying $f_{i} \leq e$ and $f_{i} q \in N$ is not empty. Let $f_{0}=\vee f_{i}$. Note that $f_{0} \in \mathcal{B}_{R}$ because of completeness of $\mathcal{B}_{R}$, and, of course, $f_{0} \leq e$. If $f_{0} q \notin N$, then, by ([4], Theorem 2.3.3), there exists $F \in M(R)$ such that $F\left(f_{0} q\right) \neq 0$ and $F(N)=0$. But, since $F\left(f_{i} q\right)=0$, we have $f_{i} e_{[\{F(q)\}]}=0$ and so $f_{i} \leq 1-e_{[\{F(q)\}]}$ for all $i$. Hence $f_{0} \leq 1-e_{[\{F(q)\}]}$, that is, $f_{0} e_{[\{F(q)\}]}=0$, which is a contradiction with $F\left(f_{0} q\right) \neq 0$. Therefore $f_{0} q$ belongs to $N$. Take $m=\left(1-f_{0}\right) q$. Let us see that $N+C q=N \oplus C m$. Indeed, for every $p \in N+C q$, we can write:

$$
p=m^{\prime}+\lambda q=m^{\prime}+\lambda f_{0} q+\lambda m \in M+C m .
$$

Moreover, if there exists $m_{0} \in N$ and $\lambda \in C$ such that

$$
m_{0}=\lambda e m=\lambda e\left(1-f_{0}\right) q,
$$

then $\lambda e q=m_{0}+\lambda e f_{0} q \in N$. Take $\mu \in C$ such that $\lambda^{2} \mu=\lambda$ and $\mu \lambda$ is an idempotent in $C$. It is clear that $\mu \lambda e q \in M$, and so $\mu \lambda e \leq f_{0}$ by maximality. Thus, $\mu \lambda e\left(1-f_{0}\right)=0$ and $\mu m_{0}=0$. Finally, note that:

$$
0=\lambda \mu m_{0}=\lambda^{2} \mu e\left(1-f_{0}\right) q=\lambda e\left(1-f_{0}\right) q=m_{0} .
$$

Thus, the sum is direct. Note that $f_{0} \in \mathcal{B}_{R}$ verifies properties a), b) and c).

As a consequence, we have the following:

Corollary 1.2 Let $M$ be a nonzero C-submodule of $R$ and $q \in R$ such that $M \subseteq C q$. Then there exists $e \in \mathcal{B}_{R}$ such that $M=$ Ceq.

Proof. If $q \in M$ take $e=1$. In other case, $M+C q=C q$. By Proposition 1.1, there is $e \in \mathcal{B}_{R}$ such that $e q \in M$ and $C q=M \oplus C(1-e) q$. Thus, $C e q \oplus C(1-e) q=M \oplus C(1-e) q$, and so, Ceq $=M$.

Note that if $p, q \in R$ then it may be that $p \in C q$ but $q \notin C p$. This forces us to make a convenient definition of set $C$-linearly independent. We will say that $n$ nonzero elements $q_{1}, q_{2}, \cdots, q_{n}$ of $R$ are C-linearly independent (or that the set $S:=\left\{q_{1}, q_{2}, \cdots, q_{n}\right\}$ is $C$-linearly independent) if, for all $\lambda_{1}, \lambda_{2}, \cdots, \lambda_{n} \in C$, $\sum \lambda_{i} q_{i}=0$ implies $\lambda_{i} q_{i}=0$ for all $i \in\{1, \cdots, n\}$, or equivalently, if the $C$-linear envelope $M$ of the subset $S$ satisfies: $\quad M=\bigoplus_{i=1}^{n} C q_{i}$. Note that for every $0 \neq q \in R$ and $e \in \mathcal{B}_{R}$, if $e q$ and $(1-e) q$ are nonzero, then 
the sets $\mathcal{S}:=\{q\}$ and $\mathcal{S}_{1}:=\{e q,(1-e) q\}$ are $C$-linearly independent and both generate the $C$-module $C q$. In general, any $C$-finitely generated $C$-module $M$ can be obtained as the $C$-linear envelope of $C$-linearly independent sets with different cardinal. In this sense, in ([4] Theorem 2.3.9. (iv)) is asserted that one can select a $C$-linearly independent set with a minimal number of generators under certain conditions. In any case, certain properties of the vector spaces remain true for the $C$-submodules: the next results, probably well-known, are obtained as a consequence of Proposition 1.1.

Corollary 1.3 Let $\left\{q_{1}, q_{2}, \cdots, q_{n}\right\}$ be a subset of $R$ and $N \varsubsetneqq M$ two C-finitely generated C-submodules of $R$ such that $M=N+\sum_{i=1}^{n} C q_{i}$. Then there are $e_{1}, e_{2}, \cdots, e_{n} \in \mathcal{B}_{R}$ such that the subset of $R$

$$
\left\{p_{1}, p_{2}, \cdots, p_{m}\right\}=\left\{\left(1-e_{1}\right) q_{1},\left(1-e_{2}\right) q_{2}, \cdots,\left(1-e_{n}\right) q_{n} \mid\left(1-e_{i}\right) q_{i} \neq 0\right\}
$$

is $C$-linearly independent, and $M=N \oplus_{j=1}^{m} C p_{j}$.

Proof. If $q_{1} \in N$, we take $e_{1}=1$. In other case, by Proposition 1.1, there exists $e_{1} \in \mathcal{B}_{R}$ such that $N+C q_{1}=N \oplus C\left(1-e_{1}\right) q_{1}$. Now, if $q_{2} \in N \oplus C\left(1-e_{1}\right) q_{1}$ then take $e_{2}=1$, and if $q_{2} \notin N \oplus C\left(1-e_{1}\right) q_{1}$ then, by Proposition 1.1, there exists $e_{2} \in \mathcal{B}_{R}$ such that $N \oplus C\left(1-e_{1} q_{1}\right)+C q_{2}=N \oplus C\left(1-e_{1}\right) q_{1} \oplus C\left(1-e_{2}\right) q_{2}$. To conclude, it is enough to repeat this procedure $n$ times.

Corollary 1.4 If $N$ is a C-finitely generated C-submodule then there exist $m \leq n$ and $p_{1}, p_{2}, \cdots, p_{m} \in N$ such that $N=\oplus_{i=1}^{m} C p_{i}$.

Proof. Let $q_{1}, q_{2}, \cdots, q_{n} \in R$ such that $N \subseteq \sum_{i=1}^{n} C q_{i}$. By Corollary 1.3 we can assume that the set $\left\{q_{1}, q_{2}, \cdots, q_{n}\right\}$ is $C$-linearly independent.

It is clear that $N+\sum_{i=1}^{n} C q_{i}=\bigoplus_{i=1}^{n} C q_{i}$. By Proposition 1.1, there exist $e_{1}, e_{2}, \cdots, e_{n} \in \mathcal{B}_{R}$ such that, for every $1 \leq j \leq n, \quad e_{j} q_{j} \in N \oplus \oplus_{i=1}^{j-1} C q_{i}$ and

$$
\bigoplus_{i=1}^{n} C q_{i}=N \oplus \bigoplus_{i=1}^{n} C\left(1-e_{i}\right) q_{i}
$$

Hence,

$$
\bigoplus_{i=1}^{n-1} C q_{i} \oplus C e_{n} q_{n} \oplus C\left(1-e_{n}\right) q_{n}=N \oplus \bigoplus_{i=1}^{n-1} C\left(1-e_{i}\right) q_{i} \oplus C\left(1-e_{n}\right) q_{n} .
$$

Therefore, $\bigoplus_{i=1}^{n-1} C q_{i} \oplus C e_{n} q_{n}=N \oplus \bigoplus_{i=1}^{n-1} C\left(1-e_{i}\right) q_{i}$. Analogously, since $e_{n} q_{n}=r_{n}^{n-2}+s$ with $r_{n}^{n-2} \in N \oplus \bigoplus_{i=1}^{n-2} C\left(1-e_{i}\right) q_{i}$ and $s \in C\left(1-e_{n-1}\right) q_{n-1}$, we have

$$
\left[\bigoplus_{i=1}^{n-2} C q_{i}+C r_{n}^{n-2}+C e_{n-1} q_{n-1}\right] \oplus C\left(1-e_{n-1}\right) q_{n-1}=\left[N \oplus \bigoplus_{i=1}^{n-2} C\left(1-e_{i}\right) q_{i}\right] \oplus C\left(1-e_{n-1}\right) q_{n-1},
$$

and so, $\bigoplus_{i=1}^{n-2} C q_{i}+C r_{n}^{n-2}+C e_{n-1} q_{n-1}=N \oplus \bigoplus_{i=1}^{n-2} C\left(1-e_{i}\right) q_{i}$.

By repeating this procedure, there are $r_{n}^{1}, r_{n-1}^{1}, \cdots, r_{2}^{1} \in N \oplus C\left(1-e_{1}\right) q_{1}$ such that

$$
\left[C q_{1}+C r_{3}^{1}+\cdots+C r_{n}^{1}+C e_{2} q_{2}\right] \oplus C\left(1-e_{2}\right) q_{2}=N \oplus\left(1-e_{1}\right) q_{1} \oplus\left(1-e_{2}\right) q_{2},
$$

and hence, $C q_{1}+C e_{2} q_{2}+C r_{3}^{1}+\cdots+C r_{n}^{1}=N \oplus C\left(1-e_{1}\right) q_{1}$. Therefore, since, $e_{2} q_{2}=r_{2}+s_{2}$ with $r_{2} \in N$ and $s_{2} \in\left(1-e_{1}\right) q_{1}$, and, for each $j>2, r_{j}^{1}=r_{j}+s_{j}$ with $r_{j} \in N$ and $s_{j} \in\left(1-e_{1}\right) q_{1}$, we deduce that

$$
\left[C e_{1} q_{1}+C r_{2}^{1}+\cdots+C r_{n}^{1}\right] \oplus C\left(1-e_{1}\right) q_{1}=N \oplus\left(1-e_{1}\right) q_{1},
$$

and so, $C e_{1} q_{1}+C r_{2}+\cdots+C r_{n}=N$. Again, by Corollary 1.3, we obtain $p_{1}, p_{2}, \cdots, p_{m} \quad C$-linear independent elements of $R$ such that $N=\bigoplus_{i=1}^{m} C p_{i}$.

Let $I \neq 0$ be a right ideal of $R$. We say that $I$ is a $\mathcal{B}_{R}$-minimal right ideal if for every nonzero right ideal $J$ of $R$ contained in $I$, there exists some $e \in \mathcal{B}_{R}$ such that $0 \neq e J=e I$. Note that if $R$ is prime then, since $C$ is a field, $\mathcal{B}_{R}=\{1\}$, and so, the concepts of $\mathcal{B}_{R}$-minimal right ideal and minimal right ideal agree. 
Recall that for a subset $S$ of $R$ the left annihilator $\{x \in R: x S=0\}$ will be denoted by $l(S)$. The right annihilator $r(S)$ is similarly defined.

Proposition 1.5 Let $I$ be a $\mathcal{B}_{R}$-minimal right ideal of $R$. Then there exists an idempotent $0 \neq q \in R$ and $e \in \mathcal{B}_{R}$ such that $e I=q R$. As a consequence $q R$ is a $\mathcal{B}_{R}$-minimal ideal of $R$.

Proof. Since $I \neq 0$ and $R$ is semiprime, $0 \neq I^{2} \subseteq I$, and hence there exists $0 \neq q^{\prime} \in I$ such that $0 \neq q^{\prime} I \subseteq I$. Note that this implies the existence of some $f \in \mathcal{B}_{R}$ such that $0 \neq f q^{\prime} I=f I$. Since $q^{\prime} \in I$, there exists $p \in I$ such that $0 \neq f q^{\prime} p=f q^{\prime}$. Note that $f q^{\prime} p^{2}=f q^{\prime} p$, and then: $f q^{\prime}\left(p^{2}-p\right)=0$, that is, $f\left(p^{2}-p\right) \in r\left(f q^{\prime}\right) \cap f I$. Since $r\left(f q^{\prime}\right)$ is a right ideal of $R$, if $r\left(f q^{\prime}\right) \cap f I \neq 0$, by minimality there exists $g \in \mathcal{B}_{R}$ such that $0 \neq g r\left(f q^{\prime}\right) \cap g f I=g I$. But, since $g N \subseteq g f I$, we have $g I=f g I=f q^{\prime} g I=0$, a contradiction. Hence, $f p^{2}=f p$ $\left(0 \neq f p\right.$ because $\left.f q^{\prime} p \neq 0\right)$. Then $0 \neq f p=f p^{2} \in f p R \subseteq f I \subseteq I$. Since $I$ is $\mathcal{B}_{R}$-minimal, there exists some $e \in \mathcal{B}_{R}$ such that $\operatorname{efp} R=e I$.

We finalized this section with a desirable result, which is similar to the well-known result for minimal right ideals (see for instance [4], Proposition 4.3.3).

Proposition 1.6 Let $q$ be an idempotent of $R$. The following assertion are equivalent:

1) $q R$ is $\mathcal{B}_{R}$-minimal right ideal of $R$.

2) For every $x \in q R q \backslash\{0\}$ there exist $z \in q R q$ and $e \in \mathcal{B}_{R}$ such that $x z=e q$.

Proof. (1) $\Rightarrow$ (2). Since $q$ is an idempotent, it is clear that $q$ is the unit of $q R q$. Take $x \in q R q \backslash\{0\}$. It is clear that $0 \neq x R=q x R \subseteq q R$, and so, since $x R$ is right ideal of $R$, there exists $f \in \mathcal{B}_{R}$ such that $f x R=f q R$. In particular, there is $z^{\prime} \in R$ such that $f x z^{\prime}=f q$. Therefore $x f q z^{\prime} q=f x z^{\prime} q=f q$.

$$
\text { (2) } \Rightarrow(1)
$$

Let $I$ be a nonzero right ideal of $R$ such that $I \subseteq q R$. Let us see that there exists $f \in \mathcal{B}_{R}$ such that $f q \in I$. Indeed, if we take $0 \neq p \in I$, by semiprimeness of $R$, there exists $q^{\prime} \in R$ such that $0 \neq p q^{\prime} p$. Note that $q p^{\prime}=p^{\prime}$ for every $p^{\prime} \in I \subseteq q R$. Consequently, $\quad p q^{\prime} q=q p q^{\prime} q$ is a nonzero element of $q R q$, and hence there are $z \in R$ and $e \in \mathcal{B}_{R}$ such that $\left(p q^{\prime} q\right)(q z q)=e q$. Therefore $e q \in p R \subseteq I$, and so, $e q R q \subseteq e I \subseteq e q R q$. Thus $e I=e q R q$.

A nonzero idempotent $q$ of $R$ is said to be $\mathcal{B}_{R}$-minimal when the above assertions are fulfilled.

\section{Theorem}

In this section we will prove a semiprime extension of [3], Theorem A.9. Concretely,

Theorem 2.1 Let $R$ be a centrally closed semiprime ring. Then $M(R)$ has a C-finite rank operator if, and only if, $R$ contains a $\mathcal{B}_{R}$-minimal idempotent $q$ such that $q R q$ is $C$-finitely generated.

We begin this proof with an another consequence of Proposition 1.1, which is an improvement of Corollary 1.2 to case $n>1$. Given a nonzero $C$-module $M C$-finitely generated, we will say that $\operatorname{dim}_{\mathcal{B}_{R}}(M)=n$ whenever

$$
n=\operatorname{Min}\left\{k \in \mathbb{N}: \exists p_{i}, p_{2}, \cdots, p_{k} \in R \backslash\{0\} \text { such that } M \subseteq \sum_{i=1}^{k} C p_{i}\right\} .
$$

Lemma 2.2 Let $M$ be a nonzero $C$-submodule of $R$ and suppose that, for every $f \in \mathcal{B}_{R}$ such that $f M \neq 0, \operatorname{dim}_{\mathcal{B}_{R}}(f M)=n>1$. If $M \subseteq \bigoplus_{i=1}^{n} C q_{i}$ for some $q_{i} \in R \backslash\{0\}$ then there exists $e \in \mathcal{B}_{R}$ such that $0 \neq e M=\bigoplus_{i=1}^{n} C_{e} q_{i}$.

Proof. It is clear that $M+\sum_{i=1}^{n} C q_{i}=\bigoplus_{i=1}^{n} C q_{i}$. By Proposition 1.1, there exist $f_{n} \in \mathcal{B}_{R}$ such that

$$
\bigoplus_{i=1}^{n} C q_{i}=\left[M+\bigoplus_{i=1}^{n-1} C q_{i}\right] \oplus C\left(1-f_{n}\right) q_{n}
$$

and $f_{n} q_{n} \in M+\bigoplus_{i=1}^{n-1} C q_{i}$, in fact, $f_{n} q_{n} \in f_{n} M+\bigoplus_{i=1}^{n-1} C f_{n} q_{i}$. Moreover,

$$
\bigoplus_{i=1}^{n-1} C q_{i} \oplus C f_{n} q_{n} \oplus C\left(1-f_{n}\right) q_{n}=\left[M+\bigoplus_{i=1}^{n-1} C q_{i}\right] \oplus C\left(1-f_{n}\right) q_{n} .
$$

Hence, 


$$
\bigoplus_{i=1}^{n-1} C q_{i} \oplus C f_{n} q_{n}=M+\bigoplus_{i=1}^{n-1} C q_{i}
$$

If $f_{n} q_{n}=0$, then

$$
\bigoplus_{i=1}^{n-1} C q_{i}=M+\bigoplus_{i=1}^{n-1} C q_{i}
$$

that is, $M \subseteq \bigoplus_{i=1}^{n-1} C q_{i}$, and this is a contradiction. Thus, $f_{n} q_{n} \neq 0$ and

$$
\bigoplus_{i=1}^{n} C f_{n} q_{i}=f_{n} M+\bigoplus_{i=1}^{n-1} C f_{n} q_{i}
$$

Note that if $f_{n} M=0$ then $0 \neq f_{n} q_{n} \in \bigoplus_{i=1}^{n-1} C f_{n} q_{i}$, which is a contradiction. By Proposition 1.1, there exist $f_{n-1} \in \mathcal{B}_{R}$ such that

$$
\bigoplus_{i=1}^{n} C f_{n} q_{i}=\left[f_{n} M+\bigoplus_{i=1}^{n-2} C f_{n} q_{i}\right] \oplus C\left(1-f_{n-1}\right) f_{n} q_{n-1}
$$

and $f_{n-1} f_{n} q_{n-1} \in f_{n} M+\bigoplus_{i=1}^{n-2} C f_{n} q_{i}$. Therefore, since $f_{n} q_{n}=p+p^{\prime}$ with $p \in f_{n} M+\bigoplus_{i=1}^{n-2} C f_{n} q_{i}$ and $p^{\prime} \in C\left(1-f_{n-1}\right) f_{n} q_{n-1}$, it is clear that

$$
\left[\bigoplus_{i=1}^{n-2} C f_{n} q_{i}+C p+C f_{n-1} f_{n} q_{n-1}\right] \oplus C\left(1-f_{n-1}\right) f_{n} q_{n-1}=\left[f_{n} M+\bigoplus_{i=1}^{n-2} C f_{n} q_{i}\right] \oplus C\left(1-f_{n-1}\right) f_{n} q_{n-1} .
$$

Hence,

$$
\bigoplus_{i=1}^{n-2} C f_{n} q_{i}+C p+C f_{n-1} f_{n} q_{n-1}=f_{n} M+\bigoplus_{i=1}^{n-2} C f_{n} q_{i}
$$

If $f_{n-1} f_{n} q_{n-1}=0$, then $f_{n} M$ is contained in $n-1$ summands, which is a contradiction. Hence, since $f_{n-1} p=f_{n-1} f_{n} q_{n}$, we have

$$
\bigoplus_{i=1}^{n} C f_{n-1} f_{n} q_{i}=f_{n-1} f_{n} M+\bigoplus_{i=1}^{n-2} C f_{n-1} f_{n} q_{i} .
$$

Note that if $f_{n-1} f_{n} M=0$, then $0 \neq f_{n-1} f_{n} q_{n-1} \in \bigoplus_{i=1}^{n-2} C f_{n-1} f_{n} q_{i}$, which is a contradiction. By repeating this procedure, we find $f_{2}, \cdots, f_{n} \in \mathcal{B}_{R}$ such that, $f_{2} \cdots f_{n} q_{2} \in f_{2} \cdots f_{n} M+C f_{2} \cdots f_{n} q_{1}, \quad 0 \neq f_{2} \cdots f_{n} M$, and

$$
\oplus_{i=1}^{n} C f_{2} \cdots f_{n} q_{i}=f_{2} \cdots f_{n} M \oplus C f_{2} \cdots f_{n} q_{1} .
$$
and,

Therefore, denoting $e_{2}=f_{2} \cdots f_{n}$, again by Proposition 1.1, there exists $f_{1} \in \mathcal{B}_{R}$ such that $f_{1} e_{2} q_{1} \in e_{2} M$

$$
\left[C f_{1} e_{2} q_{1}+C e_{2} q_{2}+\cdots+e_{2} q_{n}\right] \oplus C\left(1-f_{1}\right) e_{2} q_{1}=e_{2} M \oplus C\left(1-f_{1}\right) e_{2} q_{1}
$$

and hence,

$$
C f_{1} e_{2} q_{1}+C e_{2} q_{2}+\cdots+C e_{2} q_{n}=e_{2} M
$$

or even

$$
C f_{1} e_{2} q_{1}+C f_{1} e_{2} q_{2}+\cdots+C f_{1} e_{2} q_{n}=f_{1} e_{2} M .
$$

Of course, $0 \neq f_{1} e_{2} q_{1}$ because $\operatorname{dim}_{\mathcal{B}_{R}}\left(e_{2} M\right)=n$, and so, $0 \neq f_{1} e_{2} M$. Thus, take $e=f_{1} e_{2}$.

The next result is an immediate consequence of the Weak Density (see [4], Theorem 2.3.3). We will denote by $M_{p, q}$ the operator $L_{p} R_{q}$ for all $p, q \in R$.

Lemma 2.3 Let $p_{1}, \cdots, p_{n}, q_{1}, \cdots, q_{n} \in R$. Assume that $\left\{p_{1}, \cdots, p_{n}\right\}$ or $\left\{q_{1}, q_{2}, \cdots, q_{n}\right\}$ are C-linearly independent sets such that $\sum_{i=1}^{n} M_{p_{i}, q_{i}} \neq 0$. Then there are $1 \leq j \leq n$ and $G \in M(R)$ such that 


$$
0 \neq M_{p_{j}, G\left(q_{j}\right)}=\sum_{i=1}^{n} M_{p_{i}, G\left(q_{i}\right)} .
$$

Proof. Assume that $q_{1}, q_{2}, \cdots, q_{n} \in R$ are $C$-linearly independent. If $e_{\left[p_{i}\right]} e_{\left[q_{i}\right]}=0$ for all $i \in\{1, \cdots, n\}$ then, since $\sum_{i=1}^{n} M_{p_{i}, q_{i}}=\sum_{i=1}^{n} M_{\left.\left.p_{i}, e_{\left[p_{i}\right.}\right]_{T_{i}}\right]^{q_{i}}}$, we deduce that $\sum_{i=1}^{n} M_{p_{i}, q_{i}}=0$, is a contradiction. For simplicity, we can suppose that $e_{\left[p_{1}\right]} e_{\left[q_{1}\right]} \neq 0$. By [4] (Theorem 2.3.3), there exists $G=\sum_{j=1}^{m} M_{s_{j}, t_{j}}$ with $s_{j}, t_{j} \in R$, such that $G\left(e_{\left[p_{1}\right]} q_{1}\right) \neq 0$ and $G\left(q_{i}\right)=0$ for all $i \in\{2, \cdots, n\}$. Put $q_{1^{\prime}}=G\left(e_{\left[p_{1}\right]} q_{1}\right) \neq 0$, and note that, for every $q^{\prime} \in R$, we have:

$$
\sum_{j=1}^{m}\left(\sum_{i=1}^{n} p_{i} q^{\prime} M_{s_{j}, t_{j}}\left(q_{i}\right)\right)=\sum_{i=1}^{n} p_{i} q^{\prime} G\left(e_{\left[p_{i}\right]} q_{i}\right)=p_{1} q^{\prime} q_{1}^{\prime}
$$

As a consequence: $\sum_{i=1}^{n} M_{p_{i}, G\left(q_{i}\right)}=M_{p_{1}, q_{1^{\prime}}}=M_{p_{1}, G\left(q_{1}\right)}$. Moreover, by [4] (Corollary 2.3.10), $0 \neq M_{p_{1}, G\left(q_{1}\right)}$.

First step in the proof of Theorem

Proposition 2.4 If $M(R)$ has a $C$-finite rank operator then there are $p, q \in R$ such that $p R q$ is $C$ finitely generated.

Proof. First of all, given a nonzero operator $G \in M(R)$ with $C$-finite rank we can find an operator of the form $\sum_{i=1}^{n} M_{p_{i}, q_{i}}$, which has also $C$-finite rank. In fact, the most general form of $G$ is: $\sum L_{r_{i}} R_{s_{i}}+L_{r}+R_{s}+\alpha I d_{R}$ for some $\alpha \in \mathbb{K}$, and $r_{i}, s_{i}, r, s \in R$. We can take an element $q \in R$ such that $L_{p} G \neq 0$, because in other case we would have $G(R) \subseteq r(R)=0$, a contradiction. Analogously, there exists some $q \in R$ such that $R_{q} L_{p} G \neq 0$. Now, $F=M_{p, q} G$ is a nonzero operator with the desired form. Moreover, if $G(R)$ is $C$-finitely generated then $F(R)$ is also $C$-finitely generated. Secondly, taking in mind Corollary 1.3, we can assume without loss of generality that the set $\left\{p_{1}, p_{2}, \cdots, p_{n}\right\}$ is $C$-linearly independent. Finally, by Lemma 2.3 there are $p, q \in R$ and $H \in M(R)$ such that $0 \neq M_{p, q}=\sum_{i=1}^{n} M_{p_{i}, H\left(q_{i}\right)}$, and so, $p R q$ is also $C$-finitely generated.

Second step in the proof of Theorem is a consequence of Lemma 2.2, and its proof can be obtained from a careful reading of the proof of [4] (Lemma 6.1.4).

Proposition 2.5 Let $p, q \in R$ such that $0 \neq p R q$ is $C$-finitely generated. Then there exist a $\mathcal{B}_{R}$-minimal idempotent $q_{e} \in R$ such that $q_{e} R q_{e}$ is $C$-finitely generated.

Proof. Without loss of generality we can assume that $p=q$. Since, in other case, if we take $0 \neq r \in p R q$ then $0 \neq r R r \subseteq p R q$. Suppose further that $q R q=\sum_{i=1}^{n} C r_{i}$, for $r_{i} \in R$. By Corollary 1.3, we can assume that the sum is direct. Consider the set

$$
H:=\left\{k \in \mathbb{N}: k \leq n ; \exists q, q_{1}, \cdots, q_{k} \in R \backslash\{0\} \text { s.t } q R q=\bigoplus_{i=1}^{k} C q_{i}\right\} .
$$

It is clear that $n \in H$. Take $m$ as the minimum of $H$ and $q \in R$ such that $q R q=\oplus_{i=1}^{m} C q_{i}$ for some $q_{i} \in R$. Let $I=q R q R$. If $I=0$, then $q R q \subseteq l(R)=0$, which is a contradiction because of semiprimeness of $R$. Thus $I \neq 0$. Let $0 \neq J \subseteq I$ be a right ideal of $R$ and $0 \neq z=\sum_{i} q x_{i} q y_{i} \in J$, where $x_{i}, y_{i} \in R$. Setting $u=\sum_{i} x_{i} q y_{i}$ we note that $z=q u$. Note that if $z R q=0$ then $0=q u R q u$, a contradiction with the semiPrimeness. Take $0 \neq q^{\prime} \in z R q$, it is clear that $q^{\prime} R q^{\prime} \subseteq z R q \subseteq q R q$. Note that $M=q^{\prime} R q^{\prime}$ satisfies the hypothesis either of the Corollary 1.2 (if $m=1$ ) or of the Proposition 2.2 (if $m>1$ ), in any case, there is $e \in \mathcal{B}_{R}$ such that $0 \neq e q^{\prime} R e q^{\prime}=\oplus_{i=1}^{m} C e q_{i}=e(q R q)$. In particular, $e I=e q^{\prime} R e q^{\prime} R \subseteq e z R \subseteq J$. Therefore, $0 \neq e J=e I$, that is, $I$ is a $\mathcal{B}_{R}$-minimal right ideal of $R$. By Proposition 1.5, there exist $e \in \mathcal{B}_{R}$, and $q_{e} \in R$ such that $e I=q_{e} R$. Clearly $q_{e}=q_{e}^{2} \in e M$, and so $q_{e}=\sum_{i=1}^{n} q u_{i} q v_{i}$ where $u_{i}, v_{i} \in R$. Hence $q_{e} R q_{e} \subseteq \sum_{i=1}^{n} q R q v_{i}$ and so $q_{e} R q_{e}$ is $C$-finitely generated.

Finally, the converse is obvious.

\section{Funding}

Supported by the Junta de Andaluca Grant FQM290. 


\section{References}

[1] Martindale 3rd, W.S. (1969) Prime Rings Satisfying a Generalized Polynomial Identity. Journal of Algebra, 12, 576584. http://dx.doi.org/10.1016/0021-8693(69)90029-5

[2] Amitsur, S.A. (1972) On Rings of Quotiens. Symposia Mathematica, 8, 149-164.

[3] Bresar, M., Chebotar, M.A. and MartindalWe III, W.S. (2007) Functional Identities. Birkhauser Verlag, Basel-BostonBerlin.

[4] Beidar, K.I., Martindale III, W.S. and Mikhalev, A.V. (1996) Rings with Generalized Identities. Marcel Dekker, New York.

[5] Cabello, J.C., Cabrera, M., Rodríguez, A. and Roura, R. (2013) A Characterization of $\pi$-Complemented Rings. Communications in Algebra, 41, 3067-3079. http://dx.doi.org/10.1080/00927872.2012.672604 
Scientific Research Publishing (SCIRP) is one of the largest Open Access journal publishers. It is currently publishing more than 200 open access, online, peer-reviewed journals covering a wide range of academic disciplines. SCIRP serves the worldwide academic communities and contributes to the progress and application of science with its publication.

Other selected journals from SCIRP are listed as below. Submit your manuscript to us via either submit@scirp.org or Online Submission Portal.
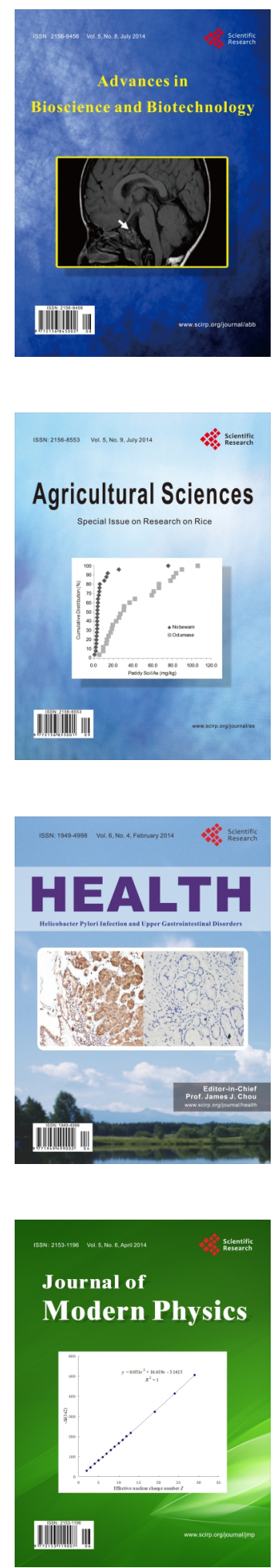
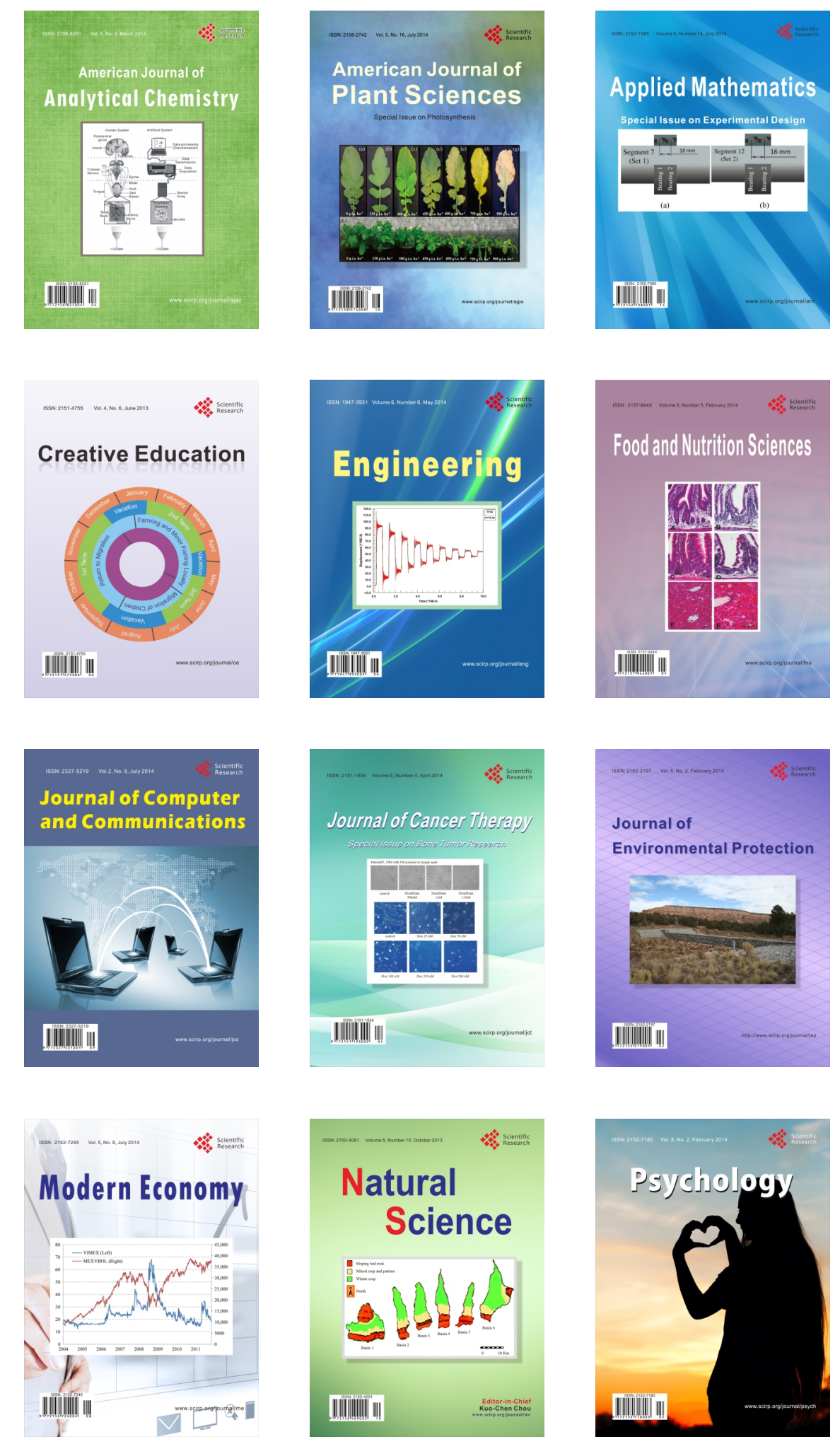